\title{
EVALUATION OF AN ONBOARD SAFETY MONITORING DEVICE IN COMMERCIAL VEHICLE OPERATIONS
}

\author{
Jeffrey S. Hickman ${ }^{1}$, Richard J. Hanowski ${ }^{1}$, and Olu Ajayi ${ }^{2}$ \\ ${ }^{1}$ Virginia Tech Transportation Institute \\ ${ }^{2}$ Federal Motor Carrier Safety Administration \\ Blacksburg, VA, USA \\ Email: jhickman@vtti.vt.edu \\ This is a work of the U.S. Government and is not subject to copyright protection in the United States.
}

\begin{abstract}
Summary: The Federal Motor Carrier Safety Administration (FMCSA) funded this project to provide an independent evaluation of DriveCam's low-cost Driving Behavior Management System (DBMS). Participating drivers drove an instrumented vehicle for 17 consecutive weeks while they made their normal, revenue-producing deliveries. During the 4-week Baseline phase, the event recorder recorded safety-related events. However, the feedback light on the event recorder was disabled and safety managers did not have access to the recorded critical incidents to provide feedback to drivers. During the 13-week Intervention phase, the feedback light on the event recorder was activated and safety managers had access to the recorded safety-related events (following the coaching protocol with drivers). Carrier A significantly reduced the mean frequency of recorded events/miles traveled from Baseline to Intervention by 37 percent $(p=0.049)$, while Carrier B significantly reduced the mean frequency of recorded events/miles traveled from Baseline to Intervention by 52.2 percent $(p=0.03)$. The results suggest the combination of onboard safety monitoring and behavioral coaching were responsible for the reduction in mean frequency of events/miles traveled at Carriers A and B.
\end{abstract}

\section{INTRODUCTION}

Motor vehicle crashes are often predictable and preventable. Yet, many drivers choose to behave in ways that put themselves and others at risk for a vehicle crash and/or serious injuries. One of the most significant studies on the factors that contribute to motor vehicle crashes was the Indiana Tri-Level Study (Treat et al., 1979). To provide insight into the factors that contribute to traffic crashes, collision data were collected on three levels to assess causal factors as being definite, probable, or possible. The study determined that 90.3 percent of the crashes involved some type of human error, such as at-risk driving behavior, inadvertent errors, and impaired states. While the vehicles in Treat et al. (1979) were predominantly light-vehicle crashes, the same relationship can be found in heavy-vehicle crashes. The recently completed Large Truck Crash Causation Study (LTCCS) assessed the causes of, and contributing factors to, crashes involving commercial motor vehicles (CMV). The LTCCS found that 87.3 percent of the critical reasons assigned to the large-truck driver were driver errors. These included 38 percent decision errors (e.g., driver drove too fast for conditions), 28.4 percent recognition errors (e.g., driver did not recognize the situation by not paying proper attention), 11.6 percent non-performance errors (e.g., driver fell asleep), and 9.2 percent performance errors (driver exercised poor directional control) (Department of Transportation, 2006). 


\section{Behavioral Approaches to Safety}

Behavioral safety programs are advantageous because they are easy to implement, easy to teach, and can be implemented in the setting where the problem occurs (Geller, 2001). Behavioral safety programs have been successfully used to increase safety-related work behaviors in a variety of organizational settings. In a review of 53 occupational safety and health studies covering various safety approaches, Gustello (1993) found behavioral safety approaches had the highest average reduction in injury rate (59.6 percent).

However, almost all prior behavioral safety research has been applied in work settings where employees can systematically observe the safe versus at-risk behavior of their coworkers. In contrast, truck and bus drivers work alone in relative isolation and thus require alternative strategies. Until recently, the primary problem has been getting quality behavioral data on driving behaviors. If behavioral approaches can be integrated with technologies that monitor driver behavior, fleet safety managers would have an effective tool for improving safety-related behaviors that occur when there is little or no opportunity for interpersonal observation and feedback.

\section{On-Board Safety Monitoring Devices}

New technologies are currently available that provide objective measures of driver behavior. These in-vehicle technologies are able to provide continuous measures on a wide variety of driving behaviors previously unavailable to fleet safety managers. The most efficacious DBMSs use in-vehicle video technology to record driver behavior. These video recordings can be used by fleet safety managers to provide feedback on safe and at-risk driving behaviors. Behavioral approaches to safety believe that modification of safe and/or at-risk driving behaviors will greatly reduce crash and injury risk. Thus, DBMSs have the potential to be used in conjunction with behavioral safety techniques to greatly reduce a variety of at-risk behaviors. The current study will provide an independent evaluation of a currently available low-cost DBMS with CMV drivers.

McGehee, Raby, Carney, Lee, and Reyes (2007) used a similar in-vehicle video technology with teen drivers. This technology provided novice teen drivers, and their parents, with a means of identifying their risky driving behaviors so that feedback could be provided to reduce future atrisk driving behaviors. Results indicated that the combination of video feedback and a graphical report card significantly decreased the rate of safety-related events in teen drivers. In the first nine weeks of the intervention, participants reduced their rate of safety-relevant events from an average of 8.6 events per 1,000 miles during baseline to 3.6 events per 1,000 miles ( 58 percent reduction). Participants further reduced the rate of events to 2.1 per 1,000 miles in the following nine weeks (76 percent reduction). The decrease from 8.6 to 2.1 events per 1,000 miles was statistically significant $(t=4.15, p=0.0007)$.

\section{METHOD}

The primary objective of this research was to provide an independent evaluation of the safety benefits of a currently available low-cost DBMS. DBMSs integrate in-vehicle video technology, 
driving performance software, and driver counseling to reduce at-risk driving behaviors. This research effort assessed the efficacy of this program and determined the improvements to driving safety (through improved driver performance) in CMV operations.

The experiment used a simple $\mathrm{A}^{4} \mathrm{~B}^{13}$ design; where " $\mathrm{A}$ " and " $\mathrm{B}$ " referred to the Baseline and Intervention phases, respectively. The superscript referred to the number of weeks in each phase (i.e., "4" refers to four weeks). During the 4-week Baseline phase, drivers drove an instrumented vehicle during their normal, revenue-producing deliveries. The event recorder was configured to record safety-related events as normal; however, the feedback light (a light on the event recorder displays each time a critical incident is recorded) was disabled and no driver counseling occurred. Immediately following the 4-week Baseline, the Intervention phase began. During the 13-week Intervention phase, drivers drove an instrumented vehicle during their normal, revenueproducing deliveries. During this time, the event recorder recorded safety-related events as normal and the DBMS program was enabled (i.e., the feedback light was activated and safety managers followed the counseling protocol). As the Virginia Tech Transportation Institute (VTTI) was the independent evaluator in this research, procedures described below were limited to those performed by VTTI.

\section{Participants and Setting}

Carrier A was a long-haul carrier located in the Southeastern U.S. that primarily delivered dry goods. A total of 50 drivers had an event recorder installed in their trucks ( 36 drivers completed data collection). A total of 46 drivers at Carrier A signed an Informed Consent Form (ICF) that allowed researchers to send questionnaires to participating drivers. The mean age of these 46 drivers was 44 years old (Range $=23$ to 61 years old). Carrier B was a local/short-haul carrier located in the Northwestern U.S. that primarily delivered beverage and paper goods. A total of 50 drivers had an event recorder installed in their trucks (41 drivers completed data collection). A total of 30 drivers at Carrier B signed an ICF that allowed researchers to send questionnaires to participating drivers. The mean age of these 30 drivers was 50 years old (Range $=27$ to 71 years old).

\section{Procedure}

Prior to the event recorders being installed in the vehicles, drivers and safety managers attended a project briefing. The project briefing lasted approximately 120 minutes and included details regarding the project, informed consent, how the DBMS technology worked, and the coaching process. Drivers indicated their interest in participating in the study by signing the ICF. Drivers who signed the ICF were asked to complete a brief Driver Demographic Questionnaire. Fifty event recorders were installed in 50 trucks at both participating carriers. Prior to the start of the 4-week Baseline phase, drivers were instructed to make their normal, revenue producing deliveries. During this time, the event recorder captured safety-related events; however, the feedback light on the event recorder was deactivated, no coaching occurred, and fleet safety managers did not have access to the data collected by the event recorder (unless a crash occurred). Prior to the start of the 13-week Intervention phase, safety manager attended a training seminar that lasted approximately 180 minutes. The safety manager training seminar included details regarding the project, informed consent, how DBMS technology worked, how to 
use the technology vendor's software, and how to "coach" drivers with the video data. Managers indicated their interest in participating in the study by signing the ICF. Safety managers who signed the ICF were asked to complete a brief Safety Manager Demographic Questionnaire. After the safety manager training seminar, the 13-week Intervention phase began. During this phase, drivers were instructed to make their normal, revenue producing deliveries. However, the feedback light on the event recorder was activated, coaching by safety managers occurred, and fleet safety managers had access to all the data collected by the event recorder during the 13week Intervention phase.

Data Collection Process. The technology vendor was responsible for all data collection. The DriveCam event recorder had two camera views: (i) driver's face view, and (ii) forward-facing view. Figure 1 displays the two camera views captured by the event recorder. The event recorder had three accelerometers (y-, x-, and z-axis) that triggered a safety-related event to be recorded. If the criterion was met or surpassed (e.g., greater than or equal to $|0.5 g|$ ) the event recorder saved 12 seconds of video (i.e., 8 seconds prior to the criterion being met or surpassed and 4 seconds after). The video and quantitative data was automatically sent to the technology vendor's headquarters in San Diego, CA via cellular transmission. Once the technology vendor received these data, they were reviewed, reduced (if necessary), and uploaded to a server. While all valid critical incidents were uploaded into the technology vendor's software for review, only those critical incidents which exceeded a certain threshold "Event Score" were requested to be reviewed with the driver. Typically, an Event Score $\geq 5$ was marked for review by a fleet safety manager; however, it was ultimately up to the fleet safety manager which events were reviewed with the drivers. Once on the server, VTTI personnel and safety managers (only during the Intervention phase) had access to the data via the technology vendor's software which was accessible via the Internet. VTTI personnel checked the software each day and recorded the frequency of critical incidents, severity of critical incidents, driving behaviors, and quantitative data.

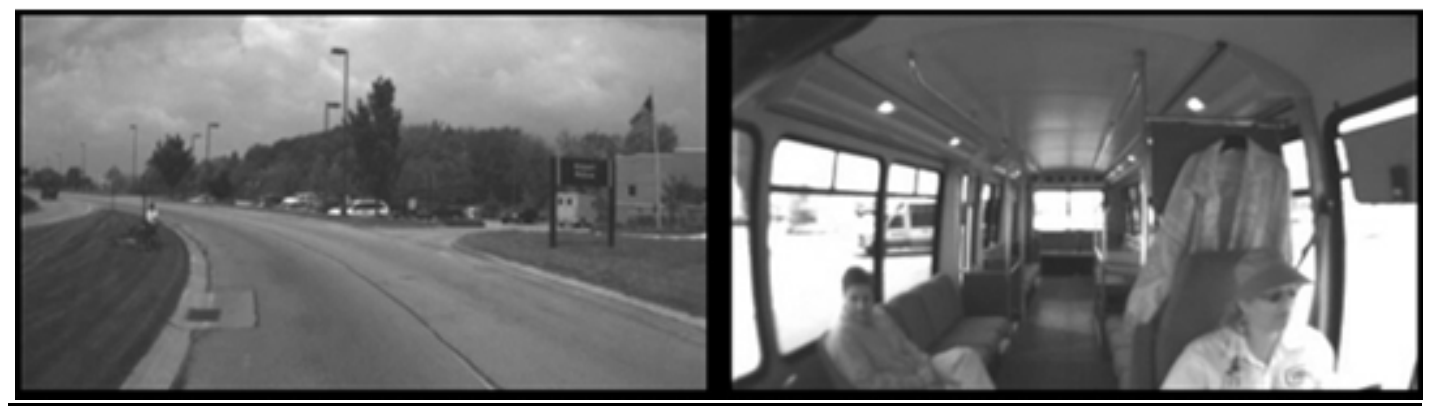

Figure 1. Front Camera View from DriveCam Event Recorder (Left) and Driver's Face View (Right)

\section{RESULTS}

\section{Carrier A}

During the 4-week Intervention phase a total of 58 valid safety-related events were captured by the event recorder ( 2 collisions and 56 risky driving events) from the remaining 36 drivers (14 drivers quit, resigned, withdrew, or had a malfunctioning event recorder). These 36 drivers drove a total of 291,869 miles during the Baseline phase. A rate was calculated to account for 
exposure (i.e., frequency of safety-related events / miles traveled). As shown in Figure 2, the mean rate of safety-related events during the Baseline phase was 0.00019 (i.e., 1.9 events per 10,000 miles). During the 13-week Intervention phase a total of 141 valid safety-related events were captured by the event recorder ( 2 collisions and 139 risky driving events). These drivers drove a total of 1,170,721 miles during the Intervention phase. As shown in Figure 2, the mean rate of safety-related events during the Intervention phase was 0.00012 (i.e., 1.2 events per 10,000 miles). A paired sample t-test found the mean rate of safety-related events during the Intervention phase ( 0.00019 events/mile traveled) was significantly lower than the mean rate of safety-related events during the Baseline phase $\left(0.00012\right.$ events/mile traveled, $t_{(35)}=1.7, p=$ 0.049). Although the 37 percent reduction in the mean rates of events from the Baseline to Intervention phase was significant, it does not indicate the magnitude of the effect. The Cohen's $d$ (or effect size) for Carrier A was 0.31. Cohen (1988) indicated that an effect size of 0.2 is considered small, 0.5 is moderate, and 0.8 is considered large. Figure 2 displays the mean rate of safety-related events per week across the 17-weeks of data collection.

Figure 2. Mean Rate of Safety-Related Events per Week across 17-Weeks of Data Collection for Carrier A

\section{Carrier B}

During the 4-week Intervention phase a total of 65 valid safety-related events were captured by the event recorder ( 1 collision and 64 risky driving events) from the remaining 41 drivers ( 9 drivers quit, resigned, or withdrew). These drivers drove a total of 162,492 miles during the Baseline phase. A rate was calculated to account for exposure (i.e., frequency of safety-related events / miles traveled). As shown in Figure 3, the mean rate of safety-related events during the Baseline phase was 0.0004 (i.e., 4.0 events per 10,000 miles). During the 13-week Intervention phase a total of 117 valid safety-related events were captured by the event recorder ( 2 collisions and 115 risky driving events). These drivers drove a total of 615,403 miles during the Intervention phase. As shown in Figure 3, the mean rate of safety-related events during the Intervention phase was 0.00019 (i.e., 1.9 events per 10,000 miles). A paired sample t-test found the mean rate of safety-related events during the Intervention phase $(0.0004$ events $/ \mathrm{mile}$ traveled $)$ 
was significantly lower than the mean rate of safety-related events during the Baseline phase $\left(0.00019\right.$ events $/$ mile traveled, $\left.t_{(40)}=1.88, p=0.03\right)$. Although the 52.2 percent reduction in the mean rates of safety-related events from the Baseline to Intervention phase was significant, it does not indicate the magnitude of the effect. The Cohen's $d$ (or effect size) for Carrier was 0.28 (small). Figure 3 displays the mean rate of safety-related events per week across the 17-weeks of data collection.

Figure 3. Mean Rate of Safety-Related Events per Week across 17-Weeks of Data Collection for Carrier B

\section{SUMMARY AND CONCLUSIONS}

In this quasi-experimental study the effectiveness of a DBMS to decrease the risky driving behaviors of local/short-haul and long-haul truck drivers was evaluated. Almost all prior behavioral safety research has targeted work behaviors in settings where employees can systematically observe the safe versus at-risk behavior of their coworkers (e.g., Geller, 1998; Krause, Hidley, and Hodson, 1996). However, employees who work in relative isolation or have little oversight from a supervisor or peer require a process where objective data can be obtained to provide feedback and coaching.

Based on a total of 1,462,590 and 777,895 miles driven at Carriers A and B, respectively, the DBMS was effective in decreasing the rate of risky driving events/mile traveled. For Carrier A, the mean rate of safety-related events/mile traveled during the Intervention phase was significantly reduced by 37 percent compared to the Baseline phase. Similarly, drivers at Carrier B significantly reduced the mean rate of safety-related events/mile traveled during the Intervention phase by 52.2 percent compared to the Baseline phase. However, the overall effects of the DBMS intervention at Carriers A and B were small ( $d=0.31$ and 0.28 , respectively).

In interpreting these results, two issues are noteworthy. First, while it appears Carrier B had superior decreases to Carrier $\mathrm{A}$ in the mean rate of safety-related events/miles traveled (based on percentage reduction), concluding differential intervention impact is risky because Carrier A 
drove more safely than Carrier B during the Baseline phase (0.00019 versus 0.0004 safetyrelated events/mile traveled). Also, Carrier A and B likely experienced different safety-related environmental conditions due to the predominate roads driven. A naturalistic study by Hanowski, Olson, Hickman, and Dingus (2005) reported that long-haul drivers typically drive on rural divided roads (e.g., highways), while local/short-haul drivers typically driver on urban divided roads.

Second, drivers were aware the instrumented vehicles were recording their driving behaviors, thus, they may have altered their performance accordingly (i.e., subject reactivity). However, it is unlikely this awareness influenced intervention impact as any reactivity to the computer tracking system should have been constant across both phases. In fact, the data obtained during the Baseline phase may have been exaggerated, resulting in a less robust effect during the Intervention phase. Indeed, an effect of reactivity to being observed is likely to be most prominent at the beginning of such procedures (Campbell, 1957).

\section{ACKNOWLEDGEMENTS}

The on-road study from which these data were collected was funded by the FMCSA under Contract DTMC75-07-D-00006 (Task Order \#1). The Task Order Manager was Olu Ajayi. The opinions expressed in the paper are those of the authors and do not necessarily represent official positions of any government agency.

\section{REFERENCES}

Campbell, D. T. (1957). Factors relevant to the validity of experiments in social settings. Psychological Bulletin, 54, 279-312.

Cohen, J. (1988). Statistical power analysis for the behavioral sciences (2nd ed.). Hillsdale, NJ: Erlbuam Associates.

Department of Transportation. (2006). Report to Congress on the Large Truck Crash Causation Study. MC-R/MC-RRA, 2006.

Geller, E. S. (1998). Understanding behavior-based safety: Step-by-step methods to improve your workplace (2nd ed.). Neenah, WI: J. J. Keller \& Associates, Inc.

Geller, E. S. (2001). The Psychology of Safety Handbook. CRC Press, Boca Raton, FL.

Guastello, S. J. (1993). Do we really know how well our occupational accident prevention programs work? Safety Science, 16, 445-463.

Hanowski, R. J., Olson, R. L., Hickman, J. S., \& Dingus, T. A. The 100-Car Naturalistic Driving Study: A Descriptive Analysis of Light Vehicle-Heavy Vehicle Interactions from the Light Vehicle Driver's Perspective, Data Analysis Results. Report\# FMCSA-RT-05-005. Federal Motor Carrier Safety Administration, Washington, DC.

Krause, T. R., Hidley, J. H., \& Hodson, S. J. (1996). The behavior-based safety process: Managing involvement for an injury-free culture (2nd ed.). New York: John Wiley \& Sons, Incorporated. 
McGehee, D. V., Raby, M., Carney, C., Lee, J. D., \& Reyes, M. L. (2007). Extending parental monitoring using an event-triggered video intervention in rural teen drivers. Journal of Safety Research, 38, 215-227.

Treat, J. R., Tumbas, N. S., McDonald, S. T., Shinar, D., Hume, R. D., Mayer, R. E., Stansifer, R. L., \& Catellan, N. J. (1979). Tri-Level Study of the Causes of Traffic Accidents: Final Report Volume I: Causal Factor Tabulations and Assessments. Institute for Research in Public Safety, Indiana University, DOT HS-805 085. 